\title{
Abdominal Aortic Aneurysms Targeted by Functionalized Polysaccharide Microparticles: a new Tool for SPECT Imaging
}

\author{
Thomas Bonnard ${ }^{1,2}$, Gonord Yang ${ }^{1}$, Anne Petiet ${ }^{3}$, Véronique Ollivier ${ }^{1}$, Oualid Haddad ${ }^{4}$, Denis Arnaud ${ }^{1}$, \\ Liliane Louedec ${ }^{1}$, Laure Bachelet-Violette ${ }^{1}$, Sidi Mohammed Derkaoui ${ }^{1}$, Didier Letourneur ${ }^{1,2}$, Cedric \\ Chauvierre $^{1}$ and Catherine Le Visage ${ }^{1 凶}$ \\ 1. Inserm, U698, Cardiovascular Bio-Engineering, X. Bichat hospital, 46 Rue H. Huchard, F-75018, Paris, France; \\ 2. Institut Galilée, Université Paris 13, Sorbonne Paris Cité, F-93430, Villetaneuse, France; \\ 3. IFR 02, UFR de Médecine, site Bichat, Université Paris Diderot, F-75018, Paris, France; \\ 4. UFR SMBH, Université Paris 13, Sorbonne Paris Cité, F-93000, Bobigny, France.
}

\section{$\square$ Corresponding author: Email: catherine.levisage@inserm.fr}

(c) Ivyspring International Publisher. This is an open-access article distributed under the terms of the Creative Commons License (http://creativecommons.org/ licenses/by-nc-nd/3.0/). Reproduction is permitted for personal, noncommercial use, provided that the article is in whole, unmodified, and properly cited.

Received: 2013.09.25; Accepted: 2013.12.16; Published: 2014.03.1I

\begin{abstract}
Aneurysm diagnostic is nowadays limited by the lack of technology that enables early detection and rupture risk prediction. New non invasive tools for molecular imaging are still required. In the present study, we present an innovative SPECT diagnostic tool for abdominal aortic aneurysm (AAA) produced from injectable polysaccharide microparticles radiolabeled with technetium $99 \mathrm{~m}$ $\left({ }^{99 \mathrm{~m}} \mathrm{Tc}\right)$ and functionalized with fucoidan, a sulfated polysaccharide with the ability to target P-Selectin. P-Selectin is a cell adhesion molecule expressed on activated endothelial cells and platelets which can be found in the thrombus of aneurysms, as well as in other vascular pathologies. Microparticles with a maximum hydrodynamic diameter of $4 \mu \mathrm{m}$ were obtained by crosslinking the polysaccharides dextran and pullulan. They were functionalized with fucoidan. In vitro interactions with human activated platelets were assessed by flow cytometry that demonstrated a specific affinity of fucoidan functionalized microparticles for P-Selectin expressed by activated platelets. For in vivo $A A A$ imaging, microparticles were radiolabeled with ${ }^{99} \mathrm{~m} \mathrm{Tc}$ and intravenously injected into healthy and AAA rats obtained by elastase perfusion through the aorta wall. Animals were scanned by SPECT imaging. A strong contrast enhancement located in the abdominal aorta of AAA rats was obtained, while no signal was obtained in healthy rats or in AAA rats after injection of non-functionalized control microparticles. Histological studies revealed that functionalized radiolabeled polysaccharide microparticles were localized in the AAA wall, in the same location where P-Selectin was expressed.

These microparticles therefore constitute a promising SPECT imaging tool for AAA and potentially for other vascular diseases characterized by P-Selectin expression. Future work will focus on validating the efficiency of the microparticles to diagnose these other pathologies and the different stages of AAA. Incorporation of a therapeutic molecule is also considered.
\end{abstract}

Key words: Fucoidan, P-Selectin, 99mTc, Ligand, Radiolabeled.

\section{Introduction}

Abdominal aortic aneurysm (AAA) is a pathological dilatation of the abdominal aorta with a mortality associated to its rupture of approximately $90 \%$
$[1,2]$. Nowadays, AAA is usually diagnosed by anatomical imaging techniques such as ultrasound, computed tomography and/or magnetic resonance 
imaging [3]. Limitations of these methods are that i) they only give anatomical and morphological information and ii) the arterial wall dilatation must be relatively advanced to clearly identify the pathology. Furthermore, it has been reported that the size measurement of the AAA is not sufficient for predicting its rupture [4]. Consequently, new non-invasive techniques enabling early identification and evaluation of the AAAs rupture risk are needed [5].

A recent strategy to fulfill these requirements is to produce injectable diagnostic tools that are able to target key molecules involved in early arterial process [3]. For this purpose, several biological markers of AAA development have been identified as potential targets of the pathology [6, 7]. Many molecular imaging probes of AAA are thus developed from various contrast agents targeted toward proteins such as elastin, collagen, matrix metalloproteinase or adhesion molecules like VCAM-1, ICAM and selectins [8-11].

In this work, we focused on adhesion molecules as they are expressed earlier in the inflammatory process than the other protein markers [12]. Among those, we selected P-Selectin which has the advantage to be present on platelets and endothelial cells on activation. The role of this key targeted molecule in AAA is not yet perfectly well established but it has been clearly identified to be involved in inflammatory cell recruitment in arterial diseases though the interaction with its counterreceptor P-Selectin Glycoprotein Ligand $1[13,14]$. For this reason, it is related with the renewal and growth of biologically active arterial thrombus and with the expansion of AAA $[15,16]$. Revealing the expression of P-Selectin with a molecular imaging tool is therefore a promising clinically relevant strategy for AAA early detection, growth prevision and rupture risk assessment. Besides, several spherical injectable polymeric particles have been developed to target P-Selectin with promising binding and/or diagnostic properties. PLGA nanoparticles conjugated with the external fraction of glycoprotein Iba showed great affinity for P-Selectin coated surfaces and for activated endothelial cell layer [17]. Van Kasteren S. et al have imaged brain diseases with carbohydrate nanoparticles functionalized with natural complex glycan ligand of selectins [18]. McAteer et al. have developed microparticles of iron oxide conjugated with P-Selectin and VCAM-1 antibodies that revealed in vivo endothelial activation on MRI scans [19].

Molecular and functional imaging of AAA employs a wide variety of imaging modalities. A large amount of magnetic resonance imaging (MRI) dedicated functionalized contrast agent including ultrasmall superparamagnetic particle of iron oxide
(USPIO) or gadolinium showed feasibility of AAA prone to rupture site identification [20]. All the other modalities are considered including ultrasound, computed tomography, optical imaging but the most promising seems to be nuclear imaging methods as they provides a highly sensitive detection of the injected radioactive imaging agent. 18F-fluorodeoxy-glucose (18F-FDG) which reveals the metabolic cells on positron emission tomography (PET) enabled identification of focal inflammatory sites in AAA which may be correlated with AAA progression and rupture risk [21, 22]. However, this method as mean to predict AAA evolution is disputed [23]. Moreover, even if PET provides a better detection sensitivity than SPECT, ${ }^{99 \mathrm{mTc}}$ is the most widely used radioisotope in nuclear medicine because of its physical characteristics: optimal gamma energy for SPECT imaging $(140 \mathrm{keV})$ and short physical half-life $(\mathrm{T}=6.01 \mathrm{~h})$, allowing a low radiation burden to patients [24]. Several diagnostic tools of the AAA have thus been developed using ${ }^{99 \mathrm{~m} T \mathrm{~T}}$ detection by SPECT. In 1976, Ryo et al. published the use of ${ }^{99 \mathrm{mTc}}$ labeled red blood cells for AAA detection [25]. Iwasaki et al. have developed in 2001 a diagnostic method for non-invasive imaging of aortic dissection using $99 \mathrm{mTc}$-labeled murine anti-smooth muscle myosin monoclonal antibody in rats [26]. In 2006, Sarda-Mantel et al. imaged luminal thrombi in murine AAA with radiolabeled annexin $\mathrm{V}$ that specifically bind to phosphatidylserine exposed to the surface of apoptic cells and activated platelets [27].

In our study, a particular interest was given to dextran and pullulan which are widely used in clinical applications [28]. An implantable biodegradable hydrogel for tissue engineering applications was obtained by crosslinking these polysaccharides [29, 30]. Since this hydrogel was not injectable, we herein developed a novel water-in-oil emulsification process combined to the crosslinking of dextran and pullulan in order to obtain injectable particles. Fucoidan, a sulfated polysaccharide derived from seaweed that happens to be an occurring mimic of sialyl Lewis $X$, the natural ligand of P-Selectin [31], was used to functionalize the particles. Our group previously demonstrated its ability to bind P-Selectin and developed a radiotracer by combining ${ }^{99 \mathrm{mTc}}$ to fucoidan $[32,33]$.

The aim of this study was to produce an efficient AAA diagnostic tool from an injectable polymeric device able to be combined with a contrast agent and to target the P-Selectin which is expressed in acute AAA. For this purpose, we have developed a microparticle system which is functionalized with fucoidan and radiolabeled with ${ }^{99 \mathrm{~m} T c \text {. We demonstrate a strong }}$ SPECT contrast enhancement located in the ab- 
dominal aorta, revealing the presence of P-Selectin inside the aneurysm of an elastase induced AAA rat model. These microparticles could have clinical uses as a SPECT diagnostic tool for early detection and progression risk assessment of AAA and potentially other arterial diseases characterized by the expression of P-Selectin.

\section{Material and Methods}

\section{Microparticles preparation}

Polysaccharide microparticles (MP) were obtained from a previously described crosslinking protocol [34] coupled to a water-in-oil emulsification process.

Pullulan (9 g, MW 200,000 g/mol, Hayashibara Inc., Okayama, Japan), dextran (3 g, MW 500,000 $\mathrm{g} / \mathrm{mol}$, Sigma Aldrich, Fallavier, France) and FITC-dextran (100 mg, MW 500,000 g/mol, Sigma Aldrich) were solubilized in $40 \mathrm{~mL}$ of purified water. To prepare functionalized microparticles (MP-Fucoidan), $1.2 \mathrm{~g}$ of fucoidan (MW 57,000 g/mol, Sigma Aldrich) was blended into the mixture. Under alkaline condition $(\mathrm{NaOH} 2.3 \mathrm{M}), 100 \mathrm{mg}$ of this aqueous solution were mixed with $30 \mu \mathrm{l}$ of trisodium trimetaphosphate $(30 \%(\mathrm{w} / \mathrm{v})$ in water, Sigma Aldrich). The whole aqueous phase was straight away slowly injected into $30 \mathrm{~mL}$ of cold $\left(-5^{\circ} \mathrm{C}\right)$ colza oil containing $1.5 \%(\mathrm{w} / \mathrm{v})$ of a surfactant mixture of Span 80 (Sigma Aldrich) and Tween 80 (Fluka, Fallavier, France) with a ratio $75 / 25$, and dispersed at 28,000 rpm for 2 min with a homogenizer (Polytron PT 3100, dispersing aggregate PT-DA 07/2EC-B101, Kinematica, Luzernerstrasse, Switzerland). Next, this emulsion was transferred to an oven $\left(50^{\circ} \mathrm{C}\right)$ wherein the crosslinking step took place for 20 minutes. The oil phase was then removed by phase separation and resulting microparticles were rinsed in PBS.

The suspension was centrifuged (BR4i, JOUAN S.A., Saint Herblain, France) for 10 minutes at 3,000 g, then the supernatant was centrifuged for 10 minutes at 5,000 g. The resulting pellet was suspended at 10 $\mathrm{mg} / \mathrm{mL}$ in saline buffer and stored at $4^{\circ} \mathrm{C}$ until use.

\section{Microparticles characterization}

The surface morphology of MP and MP-Fucoidan particles was imaged using scanning electron microscopy (SEM) (Philips XL 30 ESEM-FEG, Hilsboro, OR, USA) on dried samples coated with a thin gold layer. Mean diameter, size distribution and zeta potential were analyzed by dynamic light scattering method (NanoZS, Malvern Instruments S.A., Orsay, France). Surface sulfur presence was evidenced by energy dispersive $\mathrm{X}$-ray spectroscopy (EDX) (Philips XL 30 ESEM-FEG, Hilsboro, OR, USA) and global sulfur content was quantified by UV fluo- rescence spectroscopy (THERMO TN-TS 3000, Thermo Fisher Scientific, Pittsburgh, PA, USA) on freeze-dried samples of MP, MP-Fucoidan and plain fucoidan. Fucoidan content in MP-Fucoidan was calculated from sulfur content in MP-Fucoidan and in fucoidan.

\section{Hemolytic Toxicity Assay}

Blood from healthy volunteers was collected in sodium citrate $3.8 \%$ (w/v). Erythrocytes were separated from blood plasma by centrifugation $(800 \mathrm{~g}, 5$ $\mathrm{min})$ and resuspended at $20 \%(\mathrm{v} / \mathrm{v})$ into distilled water, which was considered as producing $100 \%$ hemolysis, and into normal saline producing no hemolysis, considered as a blank. To reproduce the in vivo parameters, the same microparticle suspensions were assessed in a corresponding amount of blood (rats of $400 \mathrm{~g}$ average weight was considered to have a total blood volume of $24 \mathrm{~mL}$ ). $5 \mu \mathrm{L}$ of MP and $5 \mu \mathrm{L}$ of MP-Fucoidan $(5 \mathrm{mg} / \mathrm{mL})$ were mixed with $500 \mu \mathrm{L}$ of erythrocytes suspensions diluted in normal saline. All preparations were incubated for $1 \mathrm{~h}$ at $37^{\circ} \mathrm{C}$ and centrifuged (3000 g, $5 \mathrm{~min}$ ). Supernatants were taken and absorbance was measured at $540 \mathrm{~nm}$. The percentage of hemolysis was determined for red blood cell samples incubated with MP, MP-Fucoidan and saline by comparing to water as $100 \%$ hemolytic sample. Results were expressed as mean values $\pm \operatorname{SEM}(n=3)$.

\section{Microparticles radiolabeling}

Technetium-99m $\left({ }^{99 m} \mathrm{Tc}\right)$ labeling required the reduction of pertechnetate by a reducing agent. Labeling of MP or MP-Fucoidan was carried out by mixing $0.030 \mathrm{~mL}$ of a $5 \mathrm{mM}$ stannous chloride solution (reducing agent), $0.5 \mathrm{~mL}$ of microparticle suspension $(10 \mathrm{mg} / \mathrm{mL}), 0.2 \mathrm{~mL}$ of $\mathrm{Na}^{+},{ }^{99 \mathrm{mTc}} \mathrm{T}_{4}$-corresponding to an activity of $370 \mathrm{MBq}$. After incubation for $1 \mathrm{~h}$ at room temperature, radiolabeled microparticles were separated from $\mathrm{Na}^{+},{ }^{99} \mathrm{mCO}_{4}{ }^{-}$excess by centrifugation $(5,000 \mathrm{~g})$. In order to determine radiolabeling efficiency, microparticle pellet and supernatant activities were measured in an activimeter (Medi 40, Medisystem, Guyancourt, France) Labeling efficiency is expressed as percentage of the ratio between radioactivity associated with the microparticles and total radioactivity.

To assess the labeling stability, the radiolabeled microparticles ( $99 \mathrm{~m} \mathrm{Tc}-\mathrm{MP}$ or $99 \mathrm{mTc}-\mathrm{MP}-F u c o i d a n$ ) were resuspended in $1 \mathrm{~mL}$ of $0.9 \% \mathrm{NaCl}$ or rat plasma, and incubated at room temperature for 3 hours. Every 60 minutes, microparticles suspension were centrifuged and radioactivity associated with particles and in the supernatant was measured $(n=3)$. Stability was expressed as a percentage of the initial labeling.

For in vivo experiments, radiolabeled micropar- 
ticles were resuspended in saline $(5 \mathrm{mg} / \mathrm{mL})$ and 200 $\mu \mathrm{L}$, corresponding to an activity of about $37 \mathrm{MBq}$, were administrated intravenously to rats.

\section{In Vitro Binding Assays}

Affinity of soluble fucoidan for P-Selectin was assessed with a BIAcore X100 (GE Healthcare, Freïburg Germany). A CM5 sensorchip was coupled with recombinant human P-Selectin and fucoidan or dextran solutions was successively injected at $0 \mathrm{M}, 100$ $\mathrm{nM}, 300 \mathrm{nM}, 1 \mu \mathrm{M}$ and $3 \mu \mathrm{M}$ at a flow rate of 30 $\mu \mathrm{L} / \mathrm{min}$. The response in resonance units (RU) was recorded as a function of time. The apparent binding affinities of fucoidan for P-Selectin were determined by analysis of the kinetic of the association assuming a 1:1 Langmuir model using BIAcore evaluation software, following a previously described protocol [33].

Affinity of fucoidan functionalized microparticles for P-Selectin expressed on the surface of activated human platelets was assessed by flow cytometry. Five $\mathrm{mL}$ of blood from healthy adult volunteers was collected in sodium citrate $3.8 \%(\mathrm{w} / \mathrm{v})$. Platelet-rich plasma (PRP) was obtained by centrifugation at $120 \mathrm{~g}$ for 15 min and platelet concentration was adjusted at $2.10^{8} / \mathrm{mL}$ with autologous platelet-poor plasma (PPP). Activated PRP was obtained by stimulation of PRP with $20 \mu \mathrm{M}$ of TRAP (thrombin receptor-activating-peptide). P-Selectin expression at the platelet surface was assessed using an anti-human CD62P-FITC $(0.11 \mathrm{mg} / \mathrm{mL}$, Ancell, Bayport, MN, USA) and its isotype-matched control. In some experiments, a non-labeled anti human CD62P (1 $\mathrm{mg} / \mathrm{mL}$, Ancell) was used to block P-Selectin in activated PRP. To evaluate the binding of microparticles to platelet P-Selectin, $5 \mu \mathrm{L}$ of non-activated PRP, activated PRP or anti P-Selectin-treated activated PRP were incubated for 20 minutes with $5 \mu \mathrm{L}$ of fluorescent (FITC) MP or MP-Fucoidan together with $5 \mu \mathrm{L}$ of PE-Cy5 Mouse Anti-Human CD41a (BD Biosciences Pharmingen, Le Pont De Claix, France) to label platelets. In addition each PRP sample was incubated with an isotype-matched control antibody.

Samples were analyzed on a LSRII flow cytometer (BD Bioscience Pharmingen), with 10,000 events collected per sample with area of double positivity reflecting the affinity of microparticles (FITC) for platelets (PE-Cy5). The data were processed with FACS DiVa software and the mean FITC fluorescence intensity (MFI) was measured in the area of double positivity. Results were presented as a ratio of MFI to the control MFI (MP incubated with PRP).

\section{In vivo arterial disease model}

The ability of the radiolabeled functionalized microparticles to target P-Selectin expression in vivo was assessed in an abdominal aortic aneurysm (AAA) experimental model in rats. All experimental procedures involving the use of rats were approved by the Animal Care and Use Committee of the Claude Bernard Institute (Paris, France). The elastase model was performed on 8 male adult Wistar rats (7 weeks, CEJ) [35]. Animals were anesthetized with pentobarbital (1 $\mu \mathrm{L} / \mathrm{g}$ body weight). Porcine pancreatic elastase (2.7 $\mathrm{mg} / \mathrm{mL}$, Sigma Aldrich) was perfused into the lumen of an isolated segment of the infrarenal abdominal aorta for 15 minutes at a rate of $2.5 \mathrm{~mL} / \mathrm{h}$.

Two weeks after the surgery, when the animals present biologically active abdominal aorta aneurysm, characterized by the presence of intraluminal thrombus [36] and the expression of P-Selectin [27, 32], rats were injected with the microparticles and imaged. Injections of $200 \mu \mathrm{L}$ of $99 \mathrm{mTc}-\mathrm{MP}$ or 99mTc-MP-Fucoidan (5 mg/mL) were performed slowly, to avoid aggregation, into the penis vein. Four healthy rats served as control experiments.

\section{SPECT/CT Scan}

Helical SPECT/CT scans were performed with 4-head camera multiplexing multipinhole camera (NanoSPECT/CT plus, Bioscan Inc, Paris, France).

Immediately, after injection of $99 \mathrm{mTc}-\mathrm{MP}$ or 99mTc-MP-Fucoidan, CT acquisition focused on the abdomen was started and 15 minutes after injection, SPECT imaging was performed in the same abdomen range. The SPECT acquisition was performed with the following parameters: helical scan with 28 projections per rotation plus circular scan at the beginning and at the end of the scan range, matrix size $=256 \times 256$, zoom 1.14 (pixel size: $1 \mathrm{~mm}^{2}$ ), correction for energy, linearity and uniformity CT data were reconstructed using filtered back projection algorithm with Ram-Lak filter in plane (voxel size $147 \times 147 \mu \mathrm{m}^{2}$ ) and slice thickness equal to $147 \mu \mathrm{m}^{2}$. SPECT data were reconstructed using HiSPECT iterative reconstruction software on PC workstation. Images were visualized using the Bioscan InVivoScope software with co-registration of SPECT and CT images.

Reconstructed slices were visually assessed in 3 planes from same stereotaxic slices (sagittal slice, coronal slice 1 centered on abdominal aorta area and coronal slice 2 centered on AAA area) with and without $\mathrm{CT}$ coregistration to determine the presence of a focal uptake in the abdominal aorta according to the model. Quantification was performed on DICOM images with a DICOM processing software (OsiriX Imaging Software, Osirix, France) by calculating the ratio between the activity (mean counts) in the AAA area and in a normal region (background) on short-axis slices. The background activity was derived from a region of interest drawn over the renal aorta avoiding renal activity. 


\section{Autoradiography, histology and immunohistochemistry}

Immediately after achievement of SPECT (60 minutes after injection) animals were sacrificed with pentobarbital overdose. Abdominal aorta aneurysms of AAA rats and healthy aorta of healthy control rats were removed, washed in $0.9 \%$ saline and weighed. Radioactivity of AAA and healthy aorta was determined by gamma counting (COBRA II - Auto Gamma, Packard, Prospect, CT, USA) and the percentage of injected activity per gram was calculated. Then, aorta samples from AAA rats and control rats were frozen and cut into $20 \mu \mathrm{m}$ thick frozen sections for autoradiography and $10 \mu \mathrm{m}$ thick frozen sections for histology and immunochemistry studies. Autoradiographic images were obtained after 12 hours exposition of fifteen slides (corresponding to about $30 \mathrm{sec}-$ tions of AAA and 15 sections of healthy aorta) using a ß-imager (Beta Imager ${ }^{\mathrm{TM}}$, Biospace Lab, Paris, France). Signal intensity values of regions of interest were assessed using a quantification sofware (M3 Vision, Biospace Lab, Paris, France). The ratio between activity (mean counts $/ \mathrm{mm}^{2}$ corrected for background) of the aneurysm sections and activity of the healthy abdominal aorta sections was calculated and compared statistically. These same sections were stained both with Masson trichrome to visualize cells, nuclei, and fibrin and with Alcian blue counter stained with nuclear red to reveal polysaccharide microparticles and cell nuclei. Immunohistochemistry studies were performed on $10 \mu \mathrm{m}$ thick sections using goat mouse anti P-Selectin $(4 \mu \mathrm{g} / \mathrm{mL}$, Cruz Biotechnology Inc., Heidelberg, Germany) as a primary antibody and $\mathrm{IgG}$ rabbit anti goat as a secondary antibody $(1 \mu \mathrm{g} / \mathrm{mL}$, Dako, Les Ulis, France) revealed by DAB enhancer (Dako) and counterstained with hematoxylin. Control sections were obtained by omitting the primary antibody. Observations were performed using light and fluorescent microscopy (Nanozoomer, Hamamatsu Photonics France SARL, Massy, France).

\section{Micro-autoradiography}

Track of internal conversion electrons emitted

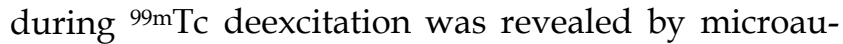
toradiography method [37]. Briefly, $10 \mu \mathrm{m}$ frozen sections of AAA and control rats were deposited on $1 \mu \mathrm{m}$ thick gelatin-coated glass slides. Five hundred $\mu \mathrm{l}$ of nuclear emulsion K5 (Ilford photo Harman technology Ltd, Ilford, United Kingdom) were poured onto section slides to obtain a $25 \mu \mathrm{m}$ thick coating. After 24 hours of exposition at $4^{\circ} \mathrm{C}$, slides were developed for 20 minutes at $15 \pm 1^{\circ} \mathrm{C}$ by Brussels formula developer $(1.8 \%(\mathrm{w} / \mathrm{v})$ sodium sulphite, $0.08 \%(\mathrm{w} / \mathrm{v})$ potassium bromide, $0.45 \%(\mathrm{w} / \mathrm{v})$ amidol and $3.5 \%$ boric acid) [38], rinsed in stop bath, fixed for 40 minutes, rinsed in tap water and finally dryed dust free, overnight at room temperature. Then, microautoradiographied slides were stained with Alcian blue in order to compare localizations of electron tracks and polysaccharide microparticles on the same sections. Observation of electrons tracks and microparticles was performed using light microscopy (Nanozoomer, Hamamatsu Photonics France SARL, Massy, France).

\section{Statistical analysis}

Flow cytometry results were analyzed statically with a one-way ANOVA with Bonferroni post tests to compare data obtained with MP-Fucoidan and a two-way ANOVA with Bonferroni post tests to compare data obtained with MP-Fucoidan and MP, performed with Graphpad Prism (GraphPad Software, San Diego, CA, USA). Other results were analyzed with unpaired Student's t-tests to compare 2 groups and one-way ANOVA with Bonferroni post test to compare 3 groups. A difference of $p<0.05$ was considered significant.

\section{Results}

\section{Characterization of polysaccharide microparticles}

Polysaccharide microparticles were prepared by a chemical crosslinking process with STMP combined with an emulsion step. Functionalized microparticles (MP-Fucoidan) were obtained by adding the sulfated polysaccharide fucoidan.

MP (Fig 1a) and MP-Fucoidan (Fig 1b) both exhibited a spherical morphology and a smooth surface as shown on scanning electronic microscopy images. Dynamic light scattering measurement revealed that all microparticles had a hydrodynamic diameter smaller than $4 \mu \mathrm{m}$ and a mean hydrodynamic diameter of $503 \pm 110 \mathrm{~nm}$ and $358 \pm 60 \mathrm{~nm}$ for MP and MP-Fucoidan, respectively (Table 1). Zeta potential was measured and microparticles prepared with fucoidan had a significantly higher electronegativity than control microparticles $(-16.2 \pm 0.8 \mathrm{mV}$ versus -9.1 $\pm 0.6 \mathrm{mV}$, respectively, $\mathrm{p}<0.05)$ (Table 1). Global sulfur content was quantified by UV fluorescence spectroscopy, using MP and plain fucoidan (in powder) as negative and positive controls, respectively. MP-Fucoidan contained $1100 \pm 400$ ppm of sulfur. Since plain fucoidan contained $6.92 \%(\mathrm{w} / \mathrm{w})$ of sulfur, we calculated the fucoidan content in MP-Fucoidan to be $1.64 \pm 0.57 \%(w / w)$. Energy-dispersive $\mathrm{X}$-ray spectroscopy analysis showed the absence of sulfur at the surface of control MP and the presence of sulfur at the surface of the MP-Fucoidan (Table 1). 

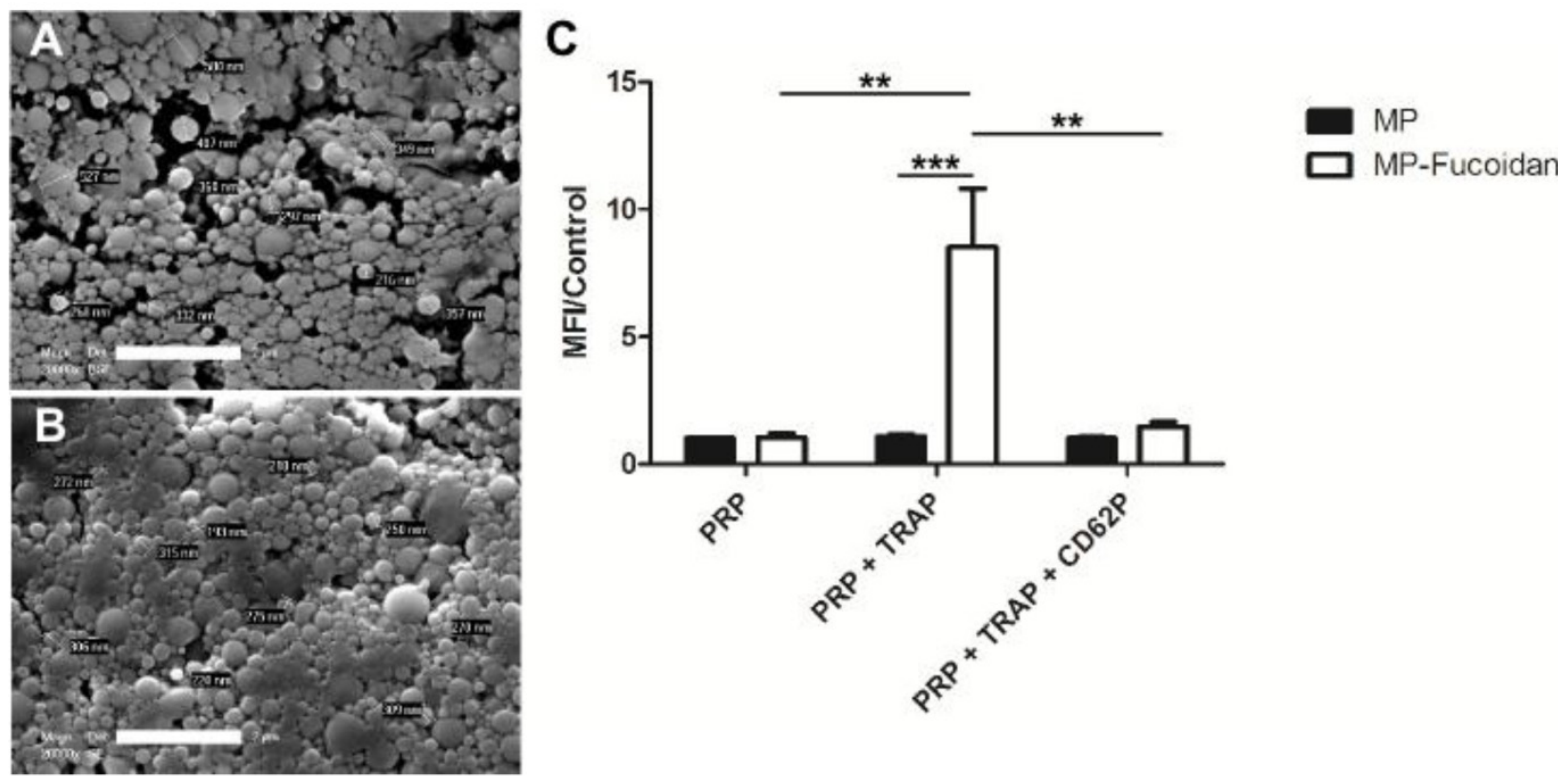

Figure I. Scanning Electron Microscopy observation of MP (a) and MP-Fucoidan (b) microparticles. Scale bar $2 \mu \mathrm{m}$. (c) Flow cytometry assessment of FITC fluorescent MP and FITC fluorescent MP-Fucoidan interaction with unactivated platelets rich plasma (PRP), platelets rich plasma activated with $20 \mu M$ TRAP (PRP + TRAP), and platelets rich plasma activated with TRAP then P-Selectin blocked with $100 \mu \mathrm{M}$ anti-CD62P (PRP + TRAP + CD62P). The mean fluorescence intensity (MFI) was measured in the area of double positivity. Results are presented as a ratio of MFI to the control (MP incubated with PRP). Results are presented as mean values $\pm \operatorname{SEM}(n=5, * * p<0.0 \mathrm{I}, * * * \mathrm{p}<0.00 \mathrm{I})$.

Table I. Characterization of microparticles. Particle size and zeta potential of polysaccharide microparticles (MP) and fucoidan functionalized microparticles (MP-Fucoidan) were measured by dynamic light scattering method $(n=5)$. Global sulfur content and fucoidan content were determined by UV fluorescence spectroscopy $(n=3)$ and surface sulfur presence was evidenced by $E D X$ technique $(n=3)$. Results are presented as mean values \pm SEM (MP versus MP-Fucoidan; $* p<0.05, * * p<0.01$ ).

\begin{tabular}{|c|c|c|c|c|c|c|c|}
\hline & \multicolumn{3}{|c|}{ Particle size } & \multirow[t]{2}{*}{ Zeta potential $(\mathrm{mV})$} & \multirow{2}{*}{$\begin{array}{l}\text { sulfur con- } \\
\text { tent } \\
\left(\mathbf{1 0}^{-3} \text { wt } \%\right)\end{array}$} & \multirow{2}{*}{$\begin{array}{l}\text { fucoidan } \\
\text { content } \\
(w t \%)\end{array}$} & \multirow{2}{*}{$\begin{array}{l}\text { sulfur } \\
\text { (EDX) }\end{array}$} \\
\hline & $\begin{array}{l}\text { Mean hydrodynamic } \\
\text { diameter (nm) }\end{array}$ & $\%<1 \mu \mathrm{m}$ & $\%<4 \mu \mathrm{m}$ & & & & \\
\hline MP & $503 \pm 110^{*}$ & $84 \pm 9$ & $100 \pm 0$ & $-9.1 \pm 0.6^{* *}$ & $<2^{*}$ & $<0.03^{*}$ & - \\
\hline MP-Fucoidan & $358 \pm 60$ & $97 \pm 2$ & $100 \pm 0$ & $-16.2 \pm 0.8$ & $113 \pm 39$ & $1.64 \pm 0.57$ & + \\
\hline
\end{tabular}

The possible toxicity induced by microparticles on red blood cells was assessed in vitro in the same conditions than in vivo administration. To reproduce these conditions, micro particle suspensions $(5 \mu \mathrm{L}$ at 5 $\mathrm{mg} / \mathrm{mL}$ ) were mixed with $500 \mu \mathrm{L}$ of erythrocytes. Hemolytic toxicity assay revealed that the MP and MP-Fucoidan suspensions lead to similarly low red blood cell lysis than normal saline $(2.00 \pm 0.31 \%$ for MP and $2.33 \pm 0.28 \%$ for MP versus $2.34 \pm 0.22 \%$ for normal saline, $\mathrm{n}=3$, no significant differences).

\section{In vitro affinity for P-Selectin}

Binding of free fucoidan to P-Selectin was analyzed by Surface Plasmon Resonance. 57 kD fucoidan and $40 \mathrm{kD}$ dextran, used as a non-sulfated polysaccharide control, were flowed on a sensorchip coated with recombinant human P-Selectin. The interaction sensorgrams revealed that fucoidan binds to P-Selectin but dextran does not (Supplementary Material: Fig. S1). Association (Ka), dissociation (Kd) and affinity (KD) constants of fucoidan for P-Selectin, calculated using a 1:1 Langmuir binding model, were $2.510^{3} \mathrm{M}, 3.210^{-5} \mathrm{M}$ and $1.210^{-8} \mathrm{M}$, respectively.

Interaction of non-functionalized microparticles with non-activated platelets (PRP), activated platelets (PRP + TRAP) and P-Selectin blocked platelets (PRP + TRAP + CD62P) was assessed and compared to the interaction of fucoidan functionalized microparticles (Fig 1c). First, a weak binding of non-functionalized microparticles for platelets was observed, whether they were incubated with activated, unactivated, or activated then blocked platelets, as shown by low values of mean fluorescence intensity (MFI). A weak binding was also noticed for fucoidan functionalized microparticles when incubated with unactivated platelets or with activated platelets blocked with anti P-Selectin, with ratios of MFI to the control MFI (MP incubated with PRP) of $1.0 \pm 0.2$ and $1.5 \pm 0.2$ respectively. In contrast, MP-Fucoidan exhibited a significantly higher MFI ratio with activated platelets, as compared to unactivated platelets or activated then blocked platelets ( $p<0.01$ in both cases). Interaction 
with platelets activated with TRAP was 8 times higher with fucoidan functionalized microparticles, as compared to non-functionalized microparticles (MFI ratio of $8.5 \pm 2.3$ versus $1.1 \pm 0.1$, respectively, $\mathrm{p}<0.001$ ).

\section{Radiolabeling of microparticles}

The ${ }^{99 \mathrm{~m} T c}$ radiolabeling yield was $90.2 \pm 2.4 \%$ on MP-Fucoidan and $91.7 \pm 1.8 \%$ on MP. It was measured after each radiolabeling and mean values were not significantly different $(n=6)$. Concerning the labeling stability (Supplementary Material: Fig. S2), the measurements revealed that radioactivity was still associated to microparticles after three hours of incubation into saline ( $89 \%$ of initial radioactivity for 99mTc-MP and $92 \%$ for $99 \mathrm{mTc}$ TP-Fucoidan). In plasma of rat, more than $75 \%$ of radioactivity was still associated after 30 minutes of incubation and more than 50 $\%$ after 1 hour for both $99 \mathrm{mTc}-\mathrm{MP}$ and 99mTc-MP-Fucoidan.

\section{In vivo SPECT imaging}

We evaluated the ability of the radiolabeled functionalized microparticles to target P-Selectin expression in vivo in an abdominal aortic aneurysm (AAA) experimental model in rats with SPECT-CT imaging (Fig 2a). While no enhancement was ob- served by SPECT 30 minutes after administration of 99mTc-MP to AAA rats or of ${ }^{99 m T c-M P-F u c o i d a n ~ t o ~}$ healthy rats, administration of $99 \mathrm{mTc}-\mathrm{MP}-\mathrm{Fucoidan}$ to AAA rats showed a strong contrast enhancement in the area of the abdominal aorta (Fig 2a, arrow).

Uptake ratios were calculated from SPECT images by the ratio of the mean intensity value of the AAA region of interest (ROI) compared to the mean intensity value of the renal aorta ROI (Fig $\mathbf{2 b}$ ). There was no uptake after administration of 99mTc-MP-Fucoidan to healthy rats (ratio $1.03 \pm 0.06$ ) or administration of $99 \mathrm{mTc}-\mathrm{MP}$ to AAA rats (ratio 1.22 \pm 0.10 ), while a significant increase of the ratio uptake with administration of $99 \mathrm{mTc}-M P-F u c o i d a n$ to AAA (ratio $3.49 \pm 0.35, p<0.01$ in both cases) was obtained.

Quantification of radioactivity was performed by gamma counting and results were expressed as a percentage of the injected activity per gram of tissue (Fig 2c). These results confirmed a significantly higher radioactivity accumulation after injection of 99mTc-MP-Fucoidan into AAA $(0.12 \pm 0.01 \%)$ as com-

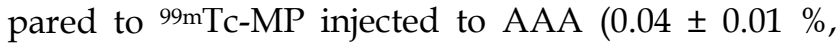
$\mathrm{p}<0.001)$ and $99 \mathrm{mTc}-\mathrm{MP}-$ Fucoidan injected to healthy rats $(0.03 \pm 0.02 \%, \mathrm{p}<0.01)$
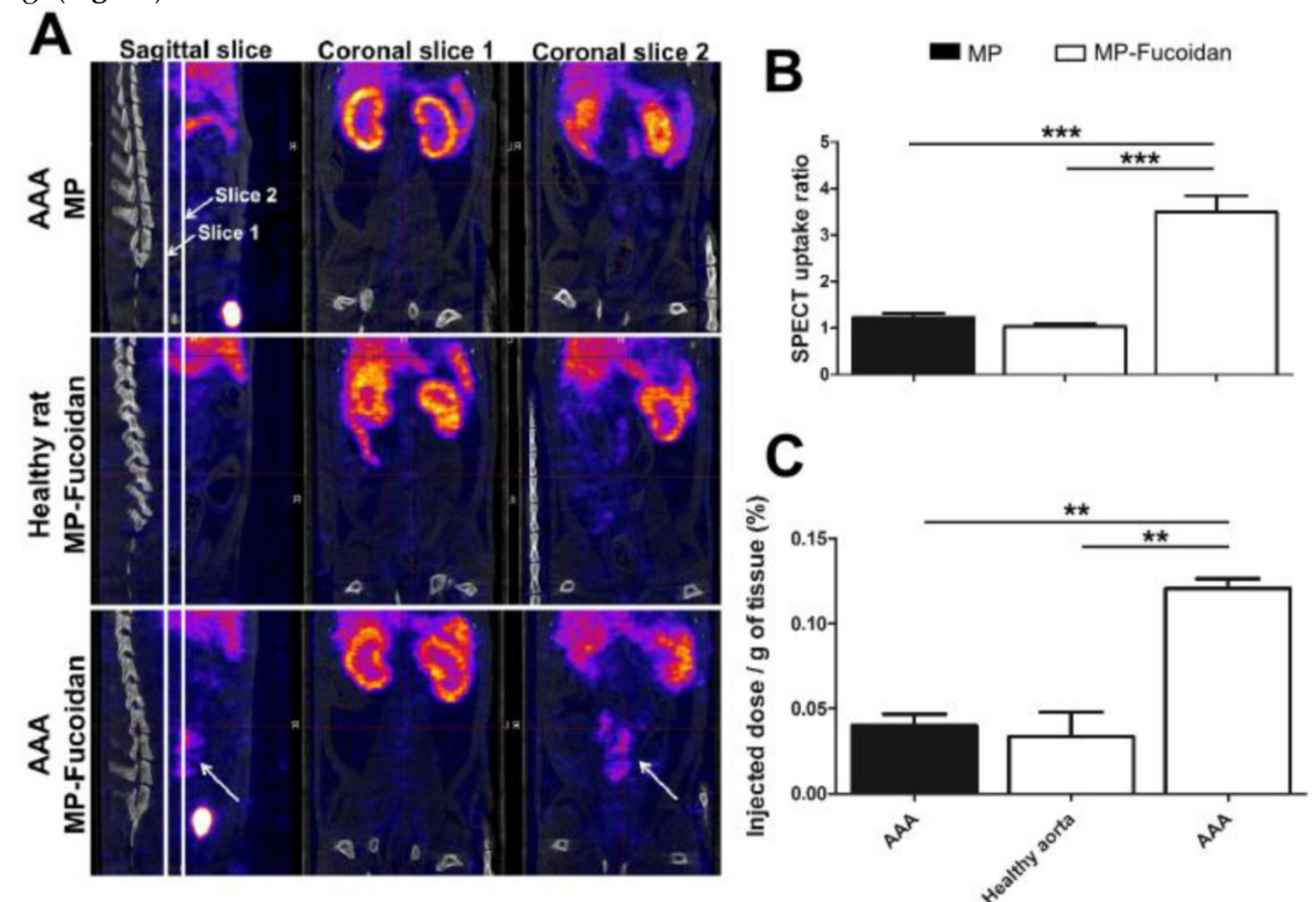

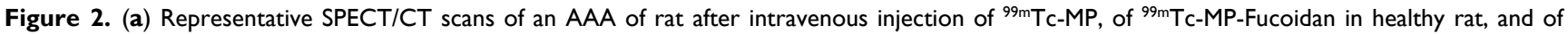
${ }^{99 m}$ Tc-MP-Fucoidan in AAA. Coronal slices I were centered on abdominal aorta areas and coronal slices 2 were centered on AAA areas. An important uptake was only noticed in the AAA area after injection of ${ }^{99 m}$ Tc-MP-Fucoidan in sagittal slice and coronal slice 2 (white arrows). (b) SPECT uptake was calculated by the ratio of the mean intensity value of the AAA region of interest (ROI) compared to the mean intensity value of the infrarenal aorta ROI $(n=3, S E M)$. (c) Accumulation of radiolabeled microparticles, expressed as a percentage of the injected dose per gram of tissue $(n=3, S E M)$. $(* *$ $p<0.0$ I, *** $\mathrm{p}<0.001)$. 


\section{Localization of ${ }^{99 \mathrm{mTC}-M P-F u c o i d a n}$ within the AAA wall}

We performed cryosections of both AAA and healthy aorta samples to analyze the fate of the functionalized microparticles. Autoradiography analysis (Supplementary Material: Fig. S3) confirmed the presence of a radioactive signal 7 times stronger in the AAA wall as compared to healthy aorta wall 60 minutes after injection of ${ }^{99 \mathrm{~m} T \mathrm{Tc}-M P-F u c o i d a n}(0.36 \pm$ $0.14 \mathrm{cpm} / \mathrm{mm}^{2}$ versus $0.05 \pm 0.02 \mathrm{cpm} / \mathrm{mm}^{2}$, respectively, $\mathrm{p}<0.05, \mathrm{n}=3$ independent experiments). Histology staining was then performed on AAA cryosections. Masson trichrome staining (Fig 3a) confirmed the presence of a thrombus and a media layer of the degraded arterial wall. In inflamed areas where cells are activated and express P-Selectin (Fig $\mathbf{3 b}$ left image and negative control without primary antibody on the right), the presence of MP-Fucoidan was clearly identified in the arterial wall using Alcian blue staining (Fig 3c). This localization was confirmed by fluorescent microscopy, where green microparticles (prepared with FITC-dextran) were observed in the arterial wall, between the thrombus and the media layer (Fig 3d). Finally, micro-autoradiography coupled to Alcian blue and nuclear red staining (Fig 3e) showed electron tracks printed into the nuclear emulsion on the same blue polysaccharide spots, confirming colo- calization of MP-Fucoidan with electron emission, i.e. radioactivity.

\section{Discussion}

In this study, we described polysaccharides microparticles functionalized with fucoidan and $99 \mathrm{mTc}$ radiolabeled.

Our group previously developed tools for diagnostic of the abdominal aortic aneurysm (AAA) intraluminal thrombus with SPECT scan, using $99 \mathrm{mTc}$ radiolabeled Annexin $\mathrm{V}$ and $99 \mathrm{mTc}$ radiolabeled $\mathrm{Fu}-$ coidan $[27,32]$. Besides their ability to image the pathology, both of these systems used small molecules and it has been reported that injectable systems that present a bigger hydrodynamic diameter have many advantages as targeting devices. Having a longer circulation time $[39,40]$ and presenting many ligands $[41,42]$ at the surface of each single object, the binding at the pathological site would improve. One other advantage working with a microparticle system instead of small molecule is that the purification steps are easy to perform by a density gradient separation method. We could thus easily remove several forms of

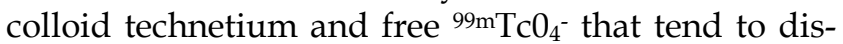
tort the biodistribution of the radiopharmaceuticals $[43,44]$. For all these reasons we have decided to develop a microparticle type diagnostic tool.

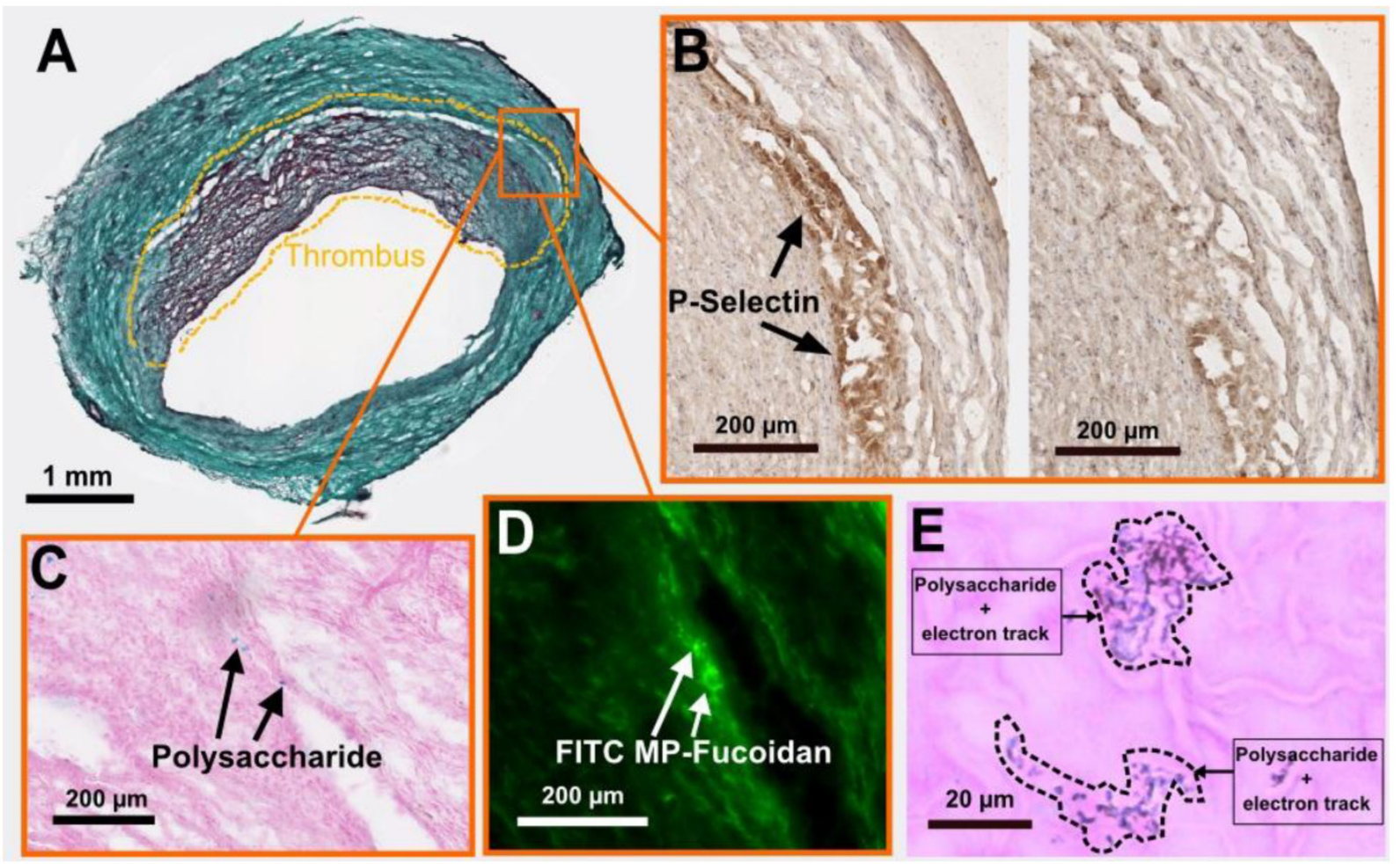

Figure 3. Histological analysis of an AAA cryosection. (a) Masson trichrome staining highlighting the thrombus area (doted lines). (b) Immunostaining of P-selectin (black arrows, left), with control section without primary antibody shown on the right. (c) Alcian blue staining (with nuclear red counterstaining) showing polysaccharide MP-Fucoidan visualized in blue localized in the arterial wall (black arrows). (d) Fluorescence microscopy observation of FITC MP-Fucoidan microparticles in the arterial wall (white arrows). (e) Micro-autoradiography on the same sections than alcian blue staining demonstrated that electron dense signals were associated with polysaccharide microparticles (dotted lines). Scale bars: I $\mu \mathrm{m}$ (a), $200 \mu \mathrm{m}$ (b,c,d) and $20 \mu \mathrm{m}$ (e). 


\section{A functionalized polymeric injectable vehicle}

The water-in-oil emulsion crosslinking method produced a reproducible distribution of microparticle size. SEM images showed that the functionalization of the microparticles did not have significant effect on surface morphology since both type of microparticles exhibit smooth surfaces. All microparticles were below $4 \mu \mathrm{m}$, though, microparticles prepared with fucoidan present a smaller hydrodynamic diameter (358 $\pm 60 \mathrm{~nm}$ versus $503 \pm 110 \mathrm{~nm}, \mathrm{p}<0.05)$. We attribute this difference to the modification in the water-in-oil emulsion parameters due to the addition of fucoidan chains in the dispersed phase. First, it altered the total polysaccharide concentration in the water phase. Secondly, the fucoidan chains have a molecular weight of $57,000 \mathrm{~g} / \mathrm{mol}$ which is small compared to dextran and pullulan chains that present a molecular weight of 500,000 g/mol and 200,000 g/mol respectively. It has been reported that decreasing the molecular weight of the polymers results in smaller particles for a water-in-oil emulsion process [45]. Finally, the fucoidan is a negatively charged polysaccharide and may modify the ionic strength of the system, known to have an influence on emulsions stability [46, 47]. The literature describes many other polymeric carriers also developed to target vascular diseases with sizes, from $100 \mathrm{~nm}$ to $5 \mu \mathrm{m}$ of mean diameter [17, 48-51]. On the one hand, nanoscale vehicles offer lower risk of vascular occlusion and avoid phagocytosis $[52,53]$. On the other hand, bigger vehicles over $500 \mathrm{~nm}$ would have a more adapted hemodynamic behaviour and have been reported as better candidates for targeting the wall in medium to large vessels relevant in several cardiovascular diseases [54-56]. Along these lines, the size distribution of our microparticles is well chosen as a compromise. They are not exclusively nanoscaled in order to improve the binding capacity and every single particle has a mean hydrodynamic diameter smaller than $4 \mu \mathrm{m}$ to avoid vessel occlusion (the smallest human capillaries are about 5 microns). Moreover, hemolytic assay demonstrated that the developed polysaccharide microparticles are not toxic, at the described concentration, for red blood cells.

For targeting the AAA, we functionalized the microparticles with fucoidan polysaccharide chains. Before adding the ligand to the system, the binding capacity of the fucoidan used in this work was studied. The SPR analyses confirmed that plain fucoidan with a molecular weight of $57,000 \mathrm{~g} / \mathrm{mol}$, has a strong affinity for P-Selectin (KD $=12 \mathrm{nM})$. It exhibited a better affinity for P-Selectin than its natural ligand does $(\mathrm{KD}=320 \mathrm{nM})$ [57].

Addition of fucoidan resulted in a significant decrease of the zeta potential $(-9.1 \pm 0.6 \mathrm{mV}$ versus $-16.2 \pm 0.8 \mathrm{mV}, \mathrm{p}<0.01)$ and an increase of the global amount of sulfur $\left(1.5 \pm 0.510^{-3} \%\right.$ wt versus $113 \pm 39$ $\left.10^{-3} \% \mathrm{wt}, \mathrm{p}<0.05\right)$. Furthermore, EDX analysis revealed sulfur presence at the surface of the microparticles. These results confirmed that fucoidan, which is an anionic sulfated polysaccharide [58], is present at the surface of the functionalized microparticles. We quantified the presence of fucoidan at $1.64 \pm 0.57 \%$ wt.

We then demonstrated that the functionalized microparticles have a strong affinity for human activated platelets. We measured by flow cytometry with functionalized microparticles a binding signal 7.7 higher than with non-functionalized control microparticles $(8.5 \pm 2.3$ MFI ratio versus $1.1 \pm 0.1$ MFI ratio, $\mathrm{p}<0.001)$. For comparison, Modery et al. assessed by receptor-specific binding studies the affinity of liposomal nanoconstructs functionalized with EWVDV peptide for activated platelets and demonstrated a 5 times increase of binding magnitude versus control [59]. We also evidenced that this affinity is specific to the P-Selectin since we measured the same level of interaction with activated-then-P-Selectin-blocked platelets than with non-activated platelets.

\section{An efficient diagnostic tool of abdominal aortic aneurysm}

The radiolabeled functionalized microparticles enabled a strong signal uptake in the aneurysm area of the AAA rats on SPECT 30 minutes after intravenous injections. The mean SPECT signal measured in the AAA areas was more than three times higher than the mean signal measured on the renal aorta whereas no enhancement was observed on SPECT of healthy rats 30 minutes after injection of the same radiolabeled functionalized microparticles (3.49 \pm 0.35 versus $1.03 \pm 0.06, \mathrm{p}<0.01)$. Rouzet et al. measured a median $99 \mathrm{mTc}$-fucoidan SPECT uptake ratio of 3.6, which means that fucoidan functionalized microparticle systems show similar diagnostic efficiency to the free radiolabeled fucoidan [32]. However, it appears that the areas of contrast uptake obtained in the AAA are larger with $99 \mathrm{mTc}-M P-F u c o i d a n$ than with 99mTc-fucoidan. In fact, when we compared the autoradiography analysis of cryosections of the harvested AAAs obtained in both cases, we evidenced that 99mTc-fucoidan brought a radioactive signal limited to the intraluminal thrombus of the AAA whereas 99mTc-MP-Fucoidan brought a signal to the whole aneurysm. Indeed, Rouzet et al. have reported that $99 \mathrm{mTc}$-fucoidan uptake and retention are more localized in the thrombus itself. The size of the diagnostic device could explain this difference. These results emphasized the reported advantages of micro and 
nanoscale carriers for targeting vessel walls. While free fucoidan molecules could not detect the injured endothelial cells which were not in the thrombus, this new SPECT diagnostic microtool would present an improved binding capacity, as compared to free fucoidan molecules, and an improved hemodynamic behavior that favors a contrast to the entire AAA injured wall. This strategy to develop a diagnostic tool of a higher hydrodynamic diameter seems therefore highly relevant.

Actually, this research seems relevant regarding the other molecular imaging tools of AAA. Indeed, our radiolabeled microparticles resulted in a higher signal uptake of AAA wall, in acute stages AAA rat models, than the ${ }^{18} \mathrm{~F}-\mathrm{FDG}$ tool in symptomatic AAA patients; uptake ratios of 3.5 was obtained with 99mTc-MP-Fucoidan versus 2.5 with ${ }^{18}$ F-FDG [21]. Our technology therefore exhibited better displays than a molecular imaging method validated by clinical trials.

Moreover, certain techniques requires a long time before reaching their targets. For instance, in magnetic resonance molecular imaging, macrophages accumulation sites imaging with USPIO and collagen imaging with targeted micelles incorporating gadolinium provides excellent contrast uptake in aneurysms, but only more than 30 hours after administration $[10,60]$. Our method with a diagnostic in the 30 minutes following the tracer injection is obviously more convenient for clinical application.

We herein validated a molecular imaging tool of P-Selectin highly effective on acute AAA, and we will, in future studies, assess its ability to detect different stages and to predict the pathology growth.

\section{A biomimetic targeting device}

Histological analysis revealed that fluorescent 99mTc-MP-Fucoidan localized inside the AAA wall. Light microscopy observation of Alcian blue stained sections and fluorescent microscopy observation of adjacent sections show microparticles in the media layer. Micro-autoradiography analysis coupled with Alcian blue staining demonstrated that the emission of electron was correlated with the presence of polysaccharide. Immunostaining revealed expression of P-Selectin in the same AAA area where 99mTc-MP-Fucoidan accumulated. One hour after injection, the functionalized microparticles developed in this study seem to be able to penetrate inside the wall of the AAA. Accumulation in the inner wall of the AAA is similar to monocyte infiltration in the inflammatory cell recruitment involved in the pathology development [61, 62]. Indeed the microparticles accumulate in the same area where macrophages have been observed on rats elastase induced AAA [63] or on mice angiotensin II induced AAA [64]. It should be noticed that leukocytes express PSGL-1 $[65,66]$ and our microparticles are functionalized with fucoidan chains that present sulfated sites that mimic the PSGL-1 anchor sites. Hence this technology could be considered as leukocyte mimetic microparticles. The property to migrate inside the arterial wall of the AAA could be a drawback for this technology and this should be taken into consideration for future work. Nevertheless, the number of microparticles seen on tissue sections is limited as compared to the quantity of leukocytes involved in the physiopathology [62]. Furthermore, this localization of the microparticles could be useful for future applications which will consist in associating a therapeutic molecule to this targeting device. Indeed, it is well known that the arterial wall degradation in aneurysm is due to a pathological proteolytic activity that occurs within the media layer and a promising strategy to treat AAA is aiming to inhibit this proteolytic activity [6]. For instance, Yoshimura et al. obtained regression of AAA with c-Jun N-terminal kinase inhibitors which reduce MMP proteolytic activity and enhance tissue repair [67]. Liao et al. demonstrated that angiotensin-converting enzyme inhibitors prohibited the degradation of medial elastin in AAA development [68]. The herein developed microparticles would act as a carrier that would bring the proteolytic inhibitor drug directly inside the AAA wall, where the therapeutic activity is needed. Besides, this targeted proteolytic action has been seriously considered for theranostic applications [69, 70]. Moreover, this functionalized system targeted toward P-Selectin will certainly exhibit a binding avidity reflecting the evolution of the pathology and therefore provide a mean to self-adjust the amount of therapeutic molecules according to the inflammation degree.

Future works will then consist in selecting the most adapted proteolytic inhibitor(s) and develop a method for its incorporation into the polysaccharide microparticles and its controlled release. As the microparticles developed are composed by reticulated polysaccharide chains, the biodegradability of a similar hydrogel being observed in a month on injured sites [71], we will propose a technology based on biodegradability for the release of the drug. Since many peptide of interest are hydrophilic and have amino groups, we can easily trap them into the microparticles or graft them with a chemical conjugation though amine bonds [72] in order to combine the therapeutic molecule of interest to the polysaccharide microparticles.

\section{Conclusion}

We hereby developed an efficient polysaccharide based SPECT diagnostic tool for the abdominal aorta 
aneurysm (AAA). In this work, we firstly described its fabrication with an emulsion-crosslinking process, confirmed its hydrodynamic diameter distribution and the presence of fucoidan ligand at the surface and characterized the radiolabeled product. We then demonstrated in vitro by flow cytometry that this fucoidan-functionalized system specifically binds to the P-Selectin expressed by human activated platelets. Finally, we showed in vivo on rats its ability to reveal AAA by SPECT imaging and we studied its fate in the AAA wall by histology experiments. Future works will consist in testing the ability of this diagnostic tool to detect the earliest stages of the AAA and in the incorporation of a therapeutic molecule.

\section{Supplementary Material}

Figs.S1 - S3. http://www.thno.org/v04p0592s1.pdf

\section{Acknowledgements}

This study was supported by Inserm and University Paris 13. T. Bonnard is a recipient of the French Ministry of Higher Education and Research Scholarship (ED Galilée, University Paris 13). The authors would like to thank F. Nadaud (UTC Compiègne, France) for SEM images, EDX analyses and technical help and P. Jame (Service Centrale d'Analyse, Villeurbane, France) for UV fluorescence spectroscopy measurements. We also acknowledge the financial support from ANR-12-EMMA-0020-01 "MicroSound", FP7 NMP-LA-2012-309820 "Nanoathero" and the Centre d'explorations fonctionnelles - Imagerie (CEFI, institut Claude Bernard).

\section{Competing Interests}

The authors have declared that no competing interest exists.

\section{References}

1. Van der Vliet JA, Boll AP. Abdominal aortic aneurysm. Lancet. 1997; 349: 863-6.

2. Nordon IM, Hinchliffe RJ, Loftus IM, Thompson MM. Pathophysiology and epidemiology of abdominal aortic aneurysms. Nat Rev Cardiol. 2011; 8: 92-102.

3. Hong H, Yang Y, Liu B, Cai W. Imaging of Abdominal Aortic Aneurysm: the present and the future. Curr Vasc Pharmacol. 2010; 8: 808-19.

4. Golledge J, Tsao PS, Dalman RL, Norman PE. Circulating markers of abdominal aortic aneurysm presence and progression. Circulation. 2008; 118: 2382-92.

5. Wassef M, Upchurch GR, Jr., Kuivaniemi H, Thompson RW, Tilson MD, 3rd. Challenges and opportunities in abdominal aortic aneurysm research. J Vasc Surg. 2007; 45: 192-8.

6. Klink A, Hyafil F, Rudd J, Faries P, Fuster V, Mallat Z, et al. Diagnostic and therapeutic strategies for small abdominal aortic aneurysms. Nat Rev Cardiol. 2011; 8: 338-47.

7. Annambhotla S, Bourgeois S, Wang X, Lin PH, Yao Q, Chen C. Recent advances in molecular mechanisms of abdominal aortic aneurysm formation. World J Surg. 2008; 32: 976-86.

8. Bazeli R, Coutard M, Duport BD, Lancelot E, Corot C, Laissy JP, et al. In vivo evaluation of a new magnetic resonance imaging contrast agent (P947) to target matrix metalloproteinases in expanding experimental abdominal aortic aneurysms. Invest Radiol. 2010; 45: 662-8.

9. Choudhury RP, Fuster V, Fayad ZA. Molecular, cellular and functional imaging of atherothrombosis. Nat Rev Drug Discov. 2004; 3: 913-25.
10. Klink A, Heynens J, Herranz B, Lobatto ME, Arias T, Sanders HM, et al. In vivo characterization of a new abdominal aortic aneurysm mouse model with conventional and molecular magnetic resonance imaging. J Am Coll Cardiol. 2011; 58: 2522-30.

11. Makowski MR, Preissel A, von Bary C, Warley A, Schachoff S, Keithan A, et al. Three-dimensional imaging of the aortic vessel wall using an elastin-specific magnetic resonance contrast agent. Invest Radiol. 2012; 47: 438-44.

12. Choudhury RP, Fisher EA. Molecular imaging in atherosclerosis, thrombosis, and vascular inflammation. Arterioscler Thromb Vasc Biol. 2009; 29: 983-91. doi:10.1161/atvbaha.108.165498.

13. Furie B, Furie BC. Role of platelet P-selectin and microparticle PSGL-1 in thrombus formation. Trends Mol Med. 2004; 10: 171-8.

14. Vandendries ER, Furie BC, Furie B. Role of P-selectin and PSGL-1 in coagulation and thrombosis. Thromb Haemost. 2004; 92: 459-66.

15. Yokoyama S, Ikeda H, Haramaki N, Yasukawa H, Murohara T, Imaizumi T. Platelet P-selectin plays an important role in arterial thrombogenesis by forming large stable platelet-leukocyte aggregates. J Am Coll Cardiol. 2005; 45: 1280-6.

16. Hannawa KK, Cho BS, Sinha I, Roelofs KJ, Myers DD, Wakefield TJ, et al. Attenuation of experimental aortic aneurysm formation in P-selectin knockout mice. Ann N Y Acad Sci. 2006; 1085: 353-9.

17. Kona S, Dong JF, Liu Y, Tan J, Nguyen KT. Biodegradable nanoparticles mimicking platelet binding as a targeted and controlled drug delivery system. Int J Pharm. 2012; 423: 516-24.

18. van Kasteren SI, Campbell SJ, Serres S, Anthony DC, Sibson NR, Davis BG. Glyconanoparticles allow pre-symptomatic in vivo imaging of brain disease. Proc Natl Acad Sci U S A. 2009; 106: 18-23.

19. McAteer MA, Mankia K, Ruparelia N, Jefferson A, Nugent HB, Stork LA, et al. A leukocyte-mimetic magnetic resonance imaging contrast agent homes rapidly to activated endothelium and tracks with atherosclerotic lesion macrophage content. Arterioscler Thromb Vasc Biol. 2012; 32: 1427-35.

20. Ramaswamy AK, Hamilton M, 2nd, Joshi RV, Kline BP, Li R, Wang P, et al. Molecular imaging of experimental abdominal aortic aneurysms. Scientific World Journal. 2013; 2013: 973150.

21. Reeps C, Essler M, Pelisek J, Seidl S, Eckstein HH, Krause BJ. Increased 18F-fluorodeoxyglucose uptake in abdominal aortic aneurysms in positron emission/computed tomography is associated with inflammation, aortic wall instability, and acute symptoms. J Vasc Surg. 2008; 48: 417-23; discussion 24.

22. Sakalihasan N, Hustinx R, Limet R. Contribution of PET scanning to the evaluation of abdominal aortic aneurysm. Semin Vasc Surg. 2004; 17: 144-53.

23. Kotze CW, Groves AM, Menezes LJ, Harvey R, Endozo R, Kayani IA, et al. What is the relationship between (1)(8)F-FDG aortic aneurysm uptake on PET/CT and future growth rate? Eur J Nucl Med Mol Imaging. 2011; 38: 1493-9.

24. Zolle I, Mazzi U, Spies H, Pietzsch HJ, Bringhammar T, Mallol J, et al. Technetium-99m pharmaceuticals: preparation and quality control in nuclear medicine. Vienna, Austria: Springer; 2007.

25. Ryo UY, Pinsky SM. Radionuclide angiography with $99 \mathrm{~m}$ technetium-RBCs. CRC Crit Rev Clin Radiol Nucl Med. 1976; 8: 107-28.

26. Iwasaki T, Aihara $Y$, Kanda $T$, Oriuchi $N$, Endo $K$, Katoh $\mathrm{H}$, et al. Immunoscintigraphy of aortic dissection with $99 \mathrm{mTc}$-labeled murine anti-smooth muscle myosin monoclonal antibody in rats. J Nucl Med. 2001; 42: 130-7.

27. Sarda-Mantel L, Coutard M, Rouzet F, Raguin O, Vrigneaud JM, Hervatin F, et al. $99 \mathrm{mTc}$-annexin-V functional imaging of luminal thrombus activity in abdominal aortic aneurysms. Arterioscler Thromb Vasc Biol. 2006; 26: 2153-9.

28. Aguilar F, Autrup H, Barlow S, Castle L, Crebelli R, Dekant W, et al. Use of lycopene as a food colour. Scientific opinion of the panel on food additives, flavourings, processing aids and materials in contact with food. Europ Food Saf Authority J. 2008;: 1-66.

29. Fricain JC, Schlaubitz S, Le Visage C, Arnault I, Derkaoui SM, Siadous R, et al. A nano-hydroxyapatite--pullulan/dextran polysaccharide composite macroporous material for bone tissue engineering. Biomaterials. 2013; 34: 2947-59.

30. Shi L, Aid R, Le Visage C, Chew SY. Biomimicking polysaccharide nanofibers promote vascular phenotypes: a potential application for vascular tissue engineering. Macromol Biosci. 2012; 12: 395-401.

31. Li B, Lu F, Wei X, Zhao R. Fucoidan: structure and bioactivity. Molecules. 2008; 13: 1671-95.

32. Rouzet F, Bachelet-Violette L, Alsac JM, Suzuki M, Meulemans A, Louedec L, et al. Radiolabeled Fucoidan as a P-Selectin Targeting Agent for In Vivo Imaging of Platelet-Rich Thrombus and Endothelial Activation. J Nucl Med. 2011; 52: 1433-40.

33. Bachelet L, Bertholon I, Lavigne D, Vassy R, Jandrot-Perrus M, Chaubet F, et al. Affinity of low molecular weight fucoidan for P-selectin triggers its binding to activated human platelets. Biochimica et Biophysica Acta (BBA) - General Subjects. 2009; 1790: 141-6.

34. Lavergne M, Derkaoui M, Delmau C, Letourneur D, Uzan G, Le Visage C. Porous polysaccharide-based scaffolds for human endothelial progenitor cells. Macromol Biosci. 2012; 12: 901-10.

35. Anidjar S, Salzmann JL, Gentric D, Lagneau P, Camilleri JP, Michel JB. Elastase-induced experimental aneurysms in rats. Circulation. 1990; 82: 973-81.

36. Coutard M, Touat Z, Houard X, Leclercq A, Michel JB. Thrombus versus wall biological activities in experimental aortic aneurysms. J Vasc Res. 2010; 47: 355-66. 
37. Robinson MS, Colas-Linhart NC, Guiraud-Vitaux FM, Petiet AM, Bok BD. Heterogeneous distribution of technetium-99m-labeled microspheres in rat lungs: microautoradiographic evidence and dosimetric consequences. J Nucl Med. 1997; 38: 650-4.

38. Limited Ht. Fact sheet ILFORD nuclear emulsions. Technical informatioin for particle physics applications. Town Lane: Ilford. 2005: 5.

39. Lammers T, Kiessling F, Hennink WE, Storm G. Nanotheranostics and image-guided drug delivery: current concepts and future directions. Mol Pharm. 2010; 7: 1899-912.

40. Svenson S. Theranostics: are we there yet? Mol Pharm. 2013; 10: 848-56.

41. Jung $\mathrm{H}$, Robison AD, Cremer PS. Multivalent ligand-receptor binding on supported lipid bilayers. J Struct Biol. 2009; 168: 90-4.

42. Wang $X$, Ramstrom $O$, Yan M. Glyconanomaterials: synthesis, characterization, and ligand presentation. Adv Mater. 2010; 22: 1946-53.

43. Vallabhajosula S, Killeen RP, Osborne JR. Altered biodistribution of radiopharmaceuticals: role of radiochemical/pharmaceutical purity, physiological, and pharmacologic factors. Semin Nucl Med. 2010; 40: 220-41.

44. Petit WA, DeLand FH, Pepper GH, Blanton L. Characterization of tin-technetium colloid in technetium-labeled albumin preparations. J Nucl Med. 1978; 19: 387-92.

45. Diez $\mathrm{S}$, Tros de Ilarduya C. Versatility of biodegradable poly(D,L-lactic-co-glycolic acid) microspheres for plasmid DNA delivery. Eur J Pharm Biopharm. 2006; 63: 188-97.

46. Gu YS, Regnier L, McClements DJ. Influence of environmental stresses on stability of oil-in-water emulsions containing droplets stabilized by beta-lactoglobulin-iota-carrageenan membranes. J Colloid Interface Sci. 2005; 286: 551-8.

47. Harnsilawat $\mathrm{T}$, Pongsawatmanit R, McClements DJ. Influence of $\mathrm{pH}$ and ionic strength on formation and stability of emulsions containing oil droplets coated by beta-lactoglobulin-alginate interfaces. Biomacromolecules. 2006; 7: 2052-8.

48. Sakhalkar HS, Hanes J, Fu J, Benavides U, Malgor R, Borruso CL, et al. Enhanced adhesion of ligand-conjugated biodegradable particles to colitic venules. FASEB J. 2005; 19: 792-4.

49. Omolola Eniola A, Hammer DA. In vitro characterization of leukocyte mimetic for targeting therapeutics to the endothelium using two receptors. Biomaterials. 2005; 26: 7136-44.

50. Simone EA, Zern BJ, Chacko AM, Mikitsh JL, Blankemeyer ER, Muro S, et al. Endothelial targeting of polymeric nanoparticles stably labeled with the PET imaging radioisotope iodine-124. Biomaterials. 2012; 33: 5406-13.

51. Song C, Labhasetwar V, Cui X, Underwood T, Levy RJ. Arterial uptake of biodegradable nanoparticles for intravascular local drug delivery: results with an acute dog model. J Control Release. 1998; 54: 201-11.

52. Champion JA, Walker A, Mitragotri S. Role of particle size in phagocytosis of polymeric microspheres. Pharm Res. 2008; 25: 1815-21. doi:10.1007/s11095-008-9562-y.

53. Eniola-Adefeso O, Heslinga MJ, Porter TM. Design of nanovectors for therapy and imaging of cardiovascular diseases. Methodist Debakey Cardiovasc J. 2012; 8: 13-7.

54. Charoenphol P, Huang RB, Eniola-Adefeso O. Potential role of size and hemodynamics in the efficacy of vascular-targeted spherical drug carriers. Biomaterials. 2010; 31: 1392-402.

55. Shinde Patil VR, Campbell CJ, Yun YH, Slack SM, Goetz DJ. Particle diameter influences adhesion under flow. Biophys J. 2001; 80: 1733-43.

56. Charoenphol P, Mocherla S, Bouis D, Namdee K, Pinsky DJ, Eniola-Adefeso O. Targeting therapeutics to the vascular wall in atherosclerosis--carrier size matters. Atherosclerosis. 2011; 217: 364-70.

57. Mehta P, Cummings RD, McEver RP. Affinity and kinetic analysis of P-selectin binding to P-selectin glycoprotein ligand-1. J Biol Chem. 1998; 273: 32506-13.

58. Dore CM, das CFAMG, Will LS, Costa TG, Sabry DA, de Souza Rego LA, et al. A sulfated polysaccharide, fucans, isolated from brown algae Sargassum vulgare with anticoagulant, antithrombotic, antioxidant and anti-inflammatory effects. Carbohydr Polym. 2013; 91: 467-75.

59. Modery CL, Ravikumar M, Wong TL, Dzuricky MJ, Durongkaveroj N, Sen Gupta A. Heteromultivalent liposomal nanoconstructs for enhanced targeting and shear-stable binding to active platelets for site-selective vascular drug delivery. Biomaterials. 2011; 32: 9504-14.

60. Sadat U, Taviani V, Patterson AJ, Young VE, Graves MJ, Teng Z, et al. Ultrasmall superparamagnetic iron oxide-enhanced magnetic resonance imaging of abdominal aortic aneurysms--a feasibility study. Eur J Vasc Endovasc Surg. 2011; 41: 167-74.

61. Hellenthal FA, Buurman WA, Wodzig WK, Schurink GW. Biomarkers of abdominal aortic aneurysm progression. Part 2: inflammation. Nat Rev Cardiol. 2009; 6: 543-52

62. Gong Y, Hart E, Shchurin A, Hoover-Plow J. Inflammatory macrophage migration requires MMP-9 activation by plasminogen in mice. J Clin Invest. 2008; 118: 3012-24.

63. Palmieri D, Pane B, Barisione C, Spinella G, Garibaldi S, Ghigliotti G, et al. Resveratrol counteracts systemic and local inflammation involved in early abdominal aortic aneurysm development. J Surg Res. 2011; 171: e237-46.

64. Yao Y, Wang Y, Zhang Y, Li Y, Sheng Z, Wen S, et al. In vivo imaging of macrophages during the early-stages of abdominal aortic aneurysm using high resolution MRI in ApoE mice. PLoS One. 2012; 7: e33523.

65. Kansas GS. Selectins and their ligands: current concepts and controversies. Blood. 1996; 88: 3259-87.
66. McEver RP. Selectin-carbohydrate interactions during inflammation and metastasis. Glycoconj J. 1997; 14: 585-91.

67. Yoshimura K, Aoki H, Ikeda Y, Fujii K, Akiyama N, Furutani A, et al. Regression of abdominal aortic aneurysm by inhibition of c-Jun N-terminal kinase. Nat Med. 2005; 11: 1330-8.

68. Liao S, Miralles M, Kelley BJ, Curci JA, Borhani M, Thompson RW. Suppression of experimental abdominal aortic aneurysms in the rat by treatment with angiotensin-converting enzyme inhibitors. J Vasc Surg. 2001; 33: $1057-64$

69. Lee S, Kim K. Protease activity: meeting its theranostic potential. Theranostics. 2012; 2 : 125-6.

70. Choi KY, Swierczewska M, Lee S, Chen X. Protease-activated drug development. Theranostics. 2012; 2: 156-78.

71. Le Visage C, Gournay O, Benguirat N, Hamidi S, Chaussumier L, Mougenot $\mathrm{N}$, et al. Mesenchymal stem cell delivery into rat infarcted myocardium using a porous polysaccharide-based scaffold: a quantitative comparison with endocardial injection. Tissue Eng Part A. 2012; 18: 35-44.

72. Sivasubramanian $\mathrm{M}$, Thambi $\mathrm{T}$, Park JH. Mineralized cyclodextrin nanoparticles for sustained protein delivery. Carbohydr Polym. 2013; 97: $643-9$ 